Research Article

\title{
On Controllability of Fractional Continuous-Time Systems
}

\author{
Mina Ghasemi $(\mathbb{D}$ and Kameleh Nassiri $\mathbb{D}$ \\ Department of Applied Mathematics, Faculty of Mathematical Sciences, University of Guilan, Rasht, Iran \\ Correspondence should be addressed to Mina Ghasemi; minaghasemi@phd.guilan.ac.ir
}

Received 5 February 2021; Revised 26 April 2021; Accepted 8 May 2021; Published 24 May 2021

Academic Editor: Adrian Neagu

Copyright ( $) 2021$ Mina Ghasemi and Kameleh Nassiri. This is an open access article distributed under the Creative Commons Attribution License, which permits unrestricted use, distribution, and reproduction in any medium, provided the original work is properly cited.

\begin{abstract}
The aim of this paper is to study the controllability of fractional systems involving the Atangana-Baleanu fractional derivative using the Caputo approach. In the first step, the solution of a linear fractional system is obtained. Then, based on the obtained solution, some necessary and sufficient conditions for the controllability of such a system will be presented. Afterwards, the controllability of a nonlinear fractional system will be analyzed, based on these results. Our tool for the presentation of the sufficient conditions of controllability in this part is Schauder fixed point theorem. In the last step, the analytical results are illustrated by numerical examples.
\end{abstract}

\section{Introduction}

Fractional calculus, with its long history, has been used in a wide range of applications. The fractional-order systems, for instance, can describe some dynamic procedures in many physical systems such as heat conduction [1] and viscoelastic materials [2] and in biology [3], bioengineering [4], energy systems [5], and economics [6]. The longmemory property of fractional calculus makes it a potent instrument for describing and designing a specific set of nonlocal dynamic trends, associated with complex systems [7]. Formerly, there have been widespread discussions in the field of dynamic properties of fractional systems. The problem of stability, for example, has been studied in [8-11].

Another important issue of fractional systems is the concept of controllability. The controllability of linear systems is established by Klamka [12]. The sufficient conditions of controllability of semilinear systems with multiple variable delays in control are formulated in [13]. BuedoFernández and Nieto [14] presented the necessary and sufficient conditions for the controllability of a linear fractional system, with constant coefficients using the Caputo fractional derivative, based on the Kalman matrix. In [15], the controllability of linear fractional dynamical systems with different order is investigated. The constrained controllability of continuous-time fractional-order control systems with multiple delays in control is considered in [16]. A computational procedure for the controllability of linear and nonlinear fractional dynamical systems of order $1<\alpha \leq 2$ is provided by Balachandran and Govindaraj [17]. Balachandran et al. [18] established a set of sufficient conditions for the controllability of nonlinear fractional dynamical systems. In [19], the controllability of nonlinear fractional delay dynamical systems with prescribed controls is considered. A variational approach to study the finite approximate controllability for Sobolev-type fractional semilinear evolution equations with nonlocal conditions in Hilbert spaces is extended by Mahmudov [20].

So far, various types of fractional derivatives such as Riemann-Liouville and Caputo have been proposed $[7,21,22]$. Derivatives such as those mentioned above can not properly model some nonlocal phenomena due to having a singular kernel. To overcome the singularity problems in fractional derivative, Caputo and Fabrizio introduced a new fractional differential operator using the exponential function as a kernel [23]. In [24], a new formulation of time fractional optimal control problems governed by Caputo-Fabrizio fractional derivative is proposed. Also, Atangana and Baleanu proposed a new 
derivative based on the Mittag-Leffler function [25]. An open discussion is ongoing about the mathematical construction of the Atangana-Baleanu fractional operator in Caputo sense (ABC). Diethelm et al. [26] showed that it is possible to solve differential equations with nonsingular kernel derivatives only when a very restrictive and unnatural assumption is made on the initial condition. Giusti [27] indicated that this operator can be expressed as an infinite series involving Riemann-Liouville integrals. The authors in [28] showed that the ABC definition cannot be useful in modeling problems such as the fractional diffusion equation because the solutions obtained for these equations do not satisfy the initial condition. Ortigueira et al. [29] showed that the models involving the generalized fractional derivative with regular kernels poorly reflect the real-world data. In responses to these criticisms, Atangana and Gómez-Aguilar [30] emphasized the need to account for a fractional calculus approach without an imposed index law and with nonsingular kernels. Furthermore, Sabatier [31] showed that the papers [26, 27] are not correct and produce the wrong conclusion on the restriction imposed by nonsingular kernels. In a comment written by Baleanu [32], it has been shown that the opinions of Ortigueira et al. [29] are not consistent. Also, Atangana and Goufo [33] presented some interesting results to clarify the mistake and lack of understanding for those writing against derivatives with nonsingular kernels. Hristov [34] investigated the underlying physical meaning of the nonsingular kernel and also presented a collection of recent applications of fractional differentiation operators with nonsingular kernels.

Motivated by the references mentioned above, we study the controllability of a nonlinear fractional system, represented as follows:

$$
\begin{aligned}
{ }^{\mathrm{ABC}} D^{\alpha} x(t) & =A x(t)+B u(t)+f(t, x(t), u(t)), \quad t \in[0, T] \\
x(0) & =x_{0},
\end{aligned}
$$

where ${ }^{\mathrm{ABC}} D^{\alpha}$ is the Atangana-Baleanu fractional derivative in Caputo sense of order $0<\alpha<1 ; x(t) \in \mathbb{R}^{n}$ and $u(t) \in \mathbb{R}^{m}$ are state and input vectors, respectively; and $A \in \mathbb{R}^{n \times n}$ and $B \in \mathbb{R}^{n \times m}$ are constant matrices. Also, $f:[0, T] \times \mathbb{R}^{n} \times$ $\mathbb{R}^{m} \longrightarrow \mathbb{R}^{n}$ and its Caputo derivative are continuous.

The following notations are used throughout this paper. For $A \in \mathbb{R}^{n \times n}, A^{*}$ denotes the matrix transpose of $A$. We assume that $I-(1-\alpha) A$ is nonsingular and $\widehat{A}=(I-(1-$ $\alpha) A)^{-1}$. We use the notations $\operatorname{Im}(A)$ and $\operatorname{Ker}(A)$, respectively, for the column space and the null space of the matrix $A$. The symbols $u_{0}$ and $f_{0}$ are used for $u(t)$ and $f(t, x(t), u(t))$, respectively, at $t=0$.

This paper is organized as follows. Section 2 presents some preliminary concepts of fractional calculus. In Section 3 , we obtain the solution of a linear fractional system and analyze the controllability of such a system. In Section 4, we establish the sufficient conditions for the controllability of a nonlinear fractional system. The obtained results are numerically confirmed in Section 5. Eventually, the conclusion is stated in Section 6 .

\section{Preliminaries}

We start with a brief overview of some mathematical preliminaries.

Definition 1. Let $\alpha \in \mathbb{R}^{+}, n-1<\alpha \leq n, n \in \mathbb{N}$, and $f$ be a suitable function. The Caputo fractional derivative of order $\alpha$ is defined as $[21,35]$

$$
{ }_{a}^{C} D_{t}^{\alpha} f(t)=\frac{1}{\Gamma(n-\alpha)} \int_{a}^{t} \frac{f^{(n)}(\tau)}{(t-\tau)^{\alpha+1-n}} \mathrm{~d} \tau .
$$

Throughout this paper $0<\alpha<1$; then,

$$
{ }_{a}^{C} D_{t}^{\alpha} f(t)=\frac{1}{\Gamma(1-\alpha)} \int_{a}^{t} \frac{f^{\prime}(\tau)}{(t-\tau)^{\alpha}} \mathrm{d} \tau .
$$

Lemma 2. Assuming that the Laplace transform $F(s)$ of the function $f$ exists, then the Laplace transform formula for the Caputo fractional derivative is [21]

$L\left({ }_{0}^{C} D_{t}^{\alpha} f(t)\right)=s^{\alpha} F(s)-\sum_{k=0}^{n-1} s^{\alpha-k-1} f^{(k)}(0), \quad n-1<\alpha \leq n$.

For $0<\alpha \leq 1$, we have

$$
L\left({ }_{0}^{C} D_{t}^{\alpha} f(t)\right)=s^{\alpha} F(s)-s^{\alpha-1} f(0) .
$$

Definition 2. The two-parameter Mittag-Leffler function, as an important function in fractional calculus, is defined as [36]

$$
E_{\alpha, \beta}(z)=\sum_{k=0}^{\infty} \frac{z^{k}}{\Gamma(\alpha k+\beta)}, \quad(\alpha>0, \beta>0) .
$$

In particular, if $\beta=1$, then

$$
E_{\alpha, 1}(z)=E_{\alpha}(z)=\sum_{k=0}^{\infty} \frac{z^{k}}{\Gamma(\alpha k+1)} .
$$

Lemma 2. The Laplace transform of the function $t^{\beta-1} E_{\alpha, \beta}\left( \pm a t^{\alpha}\right)$ is [21]

$$
L\left(t^{\beta-1} E_{\alpha, \beta}\left( \pm a t^{\alpha}\right)\right)=\frac{s^{\alpha-\beta}}{s^{\alpha} \mp a}, \quad \operatorname{Re}(s)>|a|^{(1 / \alpha)} .
$$

In particular, for $\beta=1$, equation (3) becomes

$$
L\left(E_{\alpha, 1}\left( \pm a t^{\alpha}\right)\right)=\frac{s^{\alpha-1}}{s^{\alpha} \mp a} .
$$

For Mittag-Leffler function in matrix form, similar equations are established.

Definition 3. Let $f \in H^{1}(a, b), b>a$, and $\alpha \in[0,1]$. The Atangana-Baleanu fractional derivative in Caputo sense is given as [25] 


$$
{ }_{a}^{\mathrm{ABC}} D_{t}^{\alpha} f(t)=\frac{B(\alpha)}{1-\alpha} \int_{a}^{t} f^{\prime}(x) E_{\alpha}\left(-\alpha \frac{(t-x)^{\alpha}}{1-\alpha}\right) \mathrm{d} x,
$$

where $B(\alpha)$ is a normalization function that can be any function satisfying $B(0)=B(1)=1$. Throughout this paper, we suppose $B(\alpha)=1$.

Lemma 3. Assuming that the Laplace transform $F(s)$ of the function $f$ exists, then the Laplace transform of the Atangana-Baleanu fractional derivative in Caputo sense is as follows [25]:

$$
L\left({ }_{0}^{\mathrm{ABC}} D_{t}^{\alpha} f(t)\right)=\frac{B(\alpha)}{1-\alpha} \frac{s^{\alpha} L(f(t))-s^{\alpha-1} f(0)}{s^{\alpha}+(\alpha / 1-\alpha)} .
$$

Throughout this paper, for brevity, we use ${ }^{C} D^{\alpha}$ and ${ }^{\mathrm{ABC}} D^{\alpha}$ instead of ${ }_{0}^{C} D_{t}^{\alpha}$ and ${ }_{0}^{\mathrm{ABC}} D_{t}^{\alpha}$, respectively.

\section{Linear System}

The continuous-time linear fractional system with the Atangana-Baleanu fractional derivative in Caputo sense is presented as follows:

$$
\begin{array}{r}
{ }^{\mathrm{ABC}} D^{\alpha} x(t)=A x(t)+B u(t), \quad t \in[0, T], \\
x(0)=x_{0},
\end{array}
$$

where $x(t) \in \mathbb{R}^{n}$ and $u(t) \in \mathbb{R}^{m}$ are state and input vectors, respectively, and $A \in \mathbb{R}^{n \times n}$ and $B \in \mathbb{R}^{n \times m}$ are constant matrices.

In the following theorem, we present the solution of system (12).

Theorem 1. The solution of system (12), starting from $x(0)=x_{0} \in \mathbb{R}^{n}, u(t) \in \mathbb{R}^{m}(t \geq 0)$, and assuming the existence of ${ }^{C} D^{\alpha} u(\tau)$, is

$$
\begin{aligned}
x(t)= & E_{\alpha, 1}\left(\alpha \widehat{A} A t^{\alpha}\right) x_{0}+\int_{0}^{t}(t-\tau)^{\alpha-1} E_{\alpha, \alpha}\left(\alpha \widehat{A} A(t-\tau)^{\alpha}\right) \\
& \widehat{A} B\left(\alpha u(\tau)+(1-\alpha)^{C} D^{\alpha} u(\tau)\right) \mathrm{d} \tau .
\end{aligned}
$$

Proof. Taking Laplace transform of system (12), we have

$$
\frac{s^{\alpha} X(s)-s^{\alpha-1} x_{0}}{s^{\alpha}(1-\alpha)+\alpha}=A X(s)+B U(s),
$$

which may be rewritten as

$$
(I-(1-\alpha) A) s^{\alpha} X(s)-\alpha A X(s)=s^{\alpha-1} x_{0}+(1-\alpha) B s^{\alpha} U(s)+\alpha B U(s) .
$$

Multiplying equation (15) by $\widehat{A}$, we obtain $\left(s^{\alpha} I-\alpha \widehat{A} A\right) X(s)=s^{\alpha-1} \widehat{A} x_{0}+(1-\alpha) \widehat{A} B s^{\alpha} U(s)+\alpha \widehat{A} B U(s)$.

Therefore, we can write

$$
\begin{aligned}
X(s)= & \left(s^{\alpha} I-\alpha \widehat{A} A\right)^{-1} s^{\alpha-1} \widehat{A} x_{0} \\
& +(1-\alpha)\left(s^{\alpha} I-\alpha \widehat{A} A\right)^{-1} \widehat{A} B s^{\alpha} U(s) \\
& +\alpha\left(s^{\alpha} I-\alpha \widehat{A} A\right)^{-1} \widehat{A} B U(s) .
\end{aligned}
$$

Then, adding and subtracting $(1-\alpha)\left(s^{\alpha} I-\alpha \widehat{A} A\right)^{-1}$ $\widehat{A} B s^{\alpha-1} u_{0}$ yields

$$
\begin{aligned}
X(s)= & \left(s^{\alpha} I-\alpha \widehat{A} A\right)^{-1} s^{\alpha-1} \widehat{A} x_{0} \\
& +(1-\alpha)\left(s^{\alpha} I-\alpha \widehat{A} A\right)^{-1} \widehat{A} B\left(s^{\alpha} U(s)-s^{\alpha-1} u_{0}\right) \\
& +(1-\alpha)\left(s^{\alpha} I-\alpha \widehat{A} A\right)^{-1} \widehat{A} B s^{\alpha-1} u_{0} \\
& +\alpha\left(s^{\alpha} I-\alpha \widehat{A} A\right)^{-1} \widehat{A} B U(s) .
\end{aligned}
$$

According to (5) and taking the inverse Laplace transform, we have

$$
\begin{aligned}
L^{-1}(X(s))= & L^{-1}\left(\left(s^{\alpha} I-\alpha \widehat{A} A\right)^{-1} s^{\alpha-1}\right) \widehat{A} x_{0} \\
& +(1-\alpha) L^{-1}\left(\left(s^{\alpha} I-\alpha \widehat{A} A\right)^{-1} \widehat{A} B L\left(C D^{\alpha} u(t)\right)\right) \\
& +(1-\alpha) L^{-1}\left(\left(s^{\alpha} I-\alpha \widehat{A} A\right)^{-1} \widehat{A} B s^{\alpha-1}\right) u_{0} \\
& +\alpha L^{-1}\left(\left(s^{\alpha} I-\alpha \widehat{A} A\right)^{-1} \widehat{A} B U(s)\right) .
\end{aligned}
$$

Finally, by applying the convolution theorem and equations (8), (9), and (19), we obtain

$$
\begin{aligned}
x(t) & =E_{\alpha, 1}\left(\alpha \widehat{A} A t^{\alpha}\right) \widehat{A}\left(x_{0}+(1-\alpha) B u_{0}\right) \\
& +\int_{0}^{t}(t-\tau)^{\alpha-1} E_{\alpha, \alpha}\left(\alpha \widehat{A} A(t-\tau)^{\alpha}\right) \widehat{A} B\left(\alpha u(\tau)+(1-\alpha)^{C} D^{\alpha} u(\tau)\right) \mathrm{d} \tau .
\end{aligned}
$$

Since $A x_{0}+B u_{0}=0$, we have $\widehat{A}\left(x_{0}+(1-\alpha) B u_{0}\right)=x_{0}$, and so the proof is complete.

First, we review the definition of controllability of the fractional dynamical system, in agreement with [37].

Definition 4. System (12) is controllable on $[0, T]$, if for every $x_{0}, x_{1} \in \mathbb{R}^{n}$, there exists a control $u(t) \in \mathbb{R}^{m}$ defined on $[0, T]$ such that the corresponding solution of (12) satisfies $x(T)=x_{1}$.

Corresponding to system (12), we define the controllability Gramian matrix as follows:

$$
W_{T}=\int_{0}^{T}(T-\tau)^{\alpha-1}\left[E_{\alpha, \alpha}\left(\alpha \widehat{A} A(T-\tau)^{\alpha}\right) \widehat{A} B\right]\left[E_{\alpha, \alpha}\left(\alpha \widehat{A} A(T-\tau)^{\alpha}\right) \widehat{A} B\right]^{*} \mathrm{~d} \tau .
$$


Then, we will present the controllability criteria of system (12).

Theorem 2. The continuous-time linear fractional system (12) is controllable on $[0, T]$, if and only if the controllability Gramian matrix $W_{T}$ is nonsingular.
Proof. Sufficiency: suppose that $W_{T}$ is nonsingular. For every $u_{0}$ that satisfies $A x_{0}+B u_{0}=0$, we choose the control

$$
\begin{aligned}
u(t)= & E_{\alpha, 1}\left(-\gamma t^{\alpha}\right) u_{0} \\
& +\frac{B^{*} \widehat{A}^{*}}{(1-\alpha)} \int_{0}^{t}(t-\tau)^{\alpha-1} E_{\alpha, \alpha}\left(-\gamma(t-\tau)^{\alpha}\right) E_{\alpha, \alpha}\left(\alpha A^{*} \widehat{A}^{*}(T-\tau)^{\alpha}\right) \\
& \times W_{T}^{-1}\left(x_{1}-E_{\alpha, 1}\left(\alpha \widehat{A} A T^{\alpha}\right) x_{0}\right) \mathrm{d} \tau,
\end{aligned}
$$

with $\gamma=(\alpha / 1-\alpha)$. It can be shown that $D^{\alpha} u(t)$ exists and $u(t)$ satisfies in

$$
\alpha u(t)+(1-\alpha)^{C} D^{\alpha} u(t)=B^{*} \widehat{A}^{*} E_{\alpha, \alpha}\left(\alpha A^{*} \widehat{A}^{*}(T-t)^{\alpha}\right) W_{T}^{-1}\left(x_{1}-E_{\alpha, 1}\left(\alpha \widehat{A} A T^{\alpha}\right) x_{0}\right) .
$$

Combining (13) at $t=T$ and (23), we obtain

$$
\begin{aligned}
x(T)= & E_{\alpha, 1}\left(\alpha \widehat{A} A T^{\alpha}\right) x_{0} \\
& +\int_{0}^{T}(T-\tau)^{\alpha-1} E_{\alpha, \alpha}\left(\alpha \widehat{A} A(T-\tau)^{\alpha}\right) \widehat{A} B B^{*} \widehat{A}^{*} E_{\alpha, \alpha}\left(\alpha A^{*} \widehat{A}^{*}(T-\tau)^{\alpha}\right) \\
& \times W_{T}^{-1}\left(x_{1}-E_{\alpha, 1}\left(\alpha \widehat{A} A T^{\alpha}\right) x_{0}\right) \mathrm{d} \tau \\
= & E_{\alpha, 1}\left(\alpha \widehat{A} A T^{\alpha}\right) x_{0}+W_{T} W_{T}^{-1}\left(x_{1}-E_{\alpha, 1}\left(\alpha \widehat{A} A T^{\alpha}\right) x_{0}\right)=x_{1},
\end{aligned}
$$

which shows linear system (12) is controllable.

Necessity: assume that linear system (12) is controllable. If $W_{T}$ is singular, then there exists a vector $z \neq 0$ such that $z^{*} W_{T} z=0$, which is equivalent to

$$
z^{*} \int_{0}^{T}(T-\tau)^{\alpha-1}\left[E_{\alpha, \alpha}\left(\alpha \widehat{A} A(T-\tau)^{\alpha}\right) \widehat{A} B\right]\left[E_{\alpha, \alpha}\left(\alpha \widehat{A} A(T-\tau)^{\alpha}\right) \widehat{A} B\right]^{*} \mathrm{~d} \tau z=0
$$

The preceding equation implies that

$$
z^{*} E_{\alpha, \alpha}\left(\alpha \widehat{A} A(T-\tau)^{\alpha}\right) \widehat{A} B=0, \quad \text { on }[0, T] .
$$

Let $x_{0}=\left(E_{\alpha, 1}\left(\alpha \widehat{A} A T^{\alpha}\right)\right)^{-1} z$. According to controllability assumption, there exists a control $u(t)$ on $[0, T]$ such that $x(T)=0$, which means

$$
\begin{aligned}
0= & E_{\alpha, 1}\left(\alpha \widehat{A} A T^{\alpha}\right)\left(E_{\alpha, 1}\left(\alpha \widehat{A} A T^{\alpha}\right)\right)^{-1} z \\
& +\int_{0}^{T}(T-\tau)^{\alpha-1} E_{\alpha, \alpha}\left(\alpha \widehat{A} A(T-\tau)^{\alpha}\right) \widehat{A} B\left(\alpha u(\tau)+(1-\alpha)^{C} D^{\alpha} u(\tau)\right) \mathrm{d} \tau .
\end{aligned}
$$


Multiplying equation (27) by $z^{*}$, we can write

$$
0=z^{*} z+\int_{0}^{T}(T-\tau)^{\alpha-1} z^{*} E_{\alpha, \alpha}\left(\alpha \widehat{A} A(T-\tau)^{\alpha}\right) \widehat{A} B\left(\alpha u(\tau)+(1-\alpha)^{C} D^{\alpha} u(\tau)\right) \mathrm{d} \tau
$$

Equations (26) and (28) yield $z^{*} z=0$, which is a contradiction to $z \neq 0$. Thus, $W_{T}$ is nonsingular.

Now, we present another criterion for the controllability of system (12).

Theorem 3. The continuous-time linear fractional system (12) is controllable on $[0, T]$, if and only if the Kalman matrix $K=\left[\begin{array}{llll}B & A B & \cdots & A^{n-1} B\end{array}\right]$ is full rank.
Proof. Let $K_{\mathrm{ABC}}:=\left[\widehat{A} B(\widehat{A} A) \widehat{A} B \cdots(\widehat{A} A)^{n-1} \widehat{A} B\right]$. At first, we prove that $\operatorname{Im}\left(K_{\mathrm{ABC}}\right)=\operatorname{Im}\left(W_{T}\right)$. For this purpose, we consider $R_{t}=\left\{\zeta \in \mathbb{R}^{n}\right.$ : there exists $u$ such that $\left.x(t)=\zeta\right\}$ as the set of reachable states based on system (12), with the zero initial condition. We show that for every $t>0$, $R_{t}=\operatorname{Im}\left(K_{\mathrm{ABC}}\right)=\operatorname{Im}\left(W_{T}\right)$. We will accomplish the proof in three steps.

In the first step, we prove that $R_{t} \subset \operatorname{Im}\left(K_{\mathrm{ABC}}\right)$. Let $\zeta \in R_{t}$ be every reachable state. Hence, there exists a control $u(t)$ such that

$$
\zeta=\int_{0}^{t}(t-\tau)^{\alpha-1} E_{\alpha, \alpha}\left(\alpha \widehat{A} A(t-\tau)^{\alpha}\right) \widehat{A} B\left(\alpha u(\tau)+(1-\alpha)^{C} D^{\alpha} u(\tau)\right) \mathrm{d} \tau
$$

which is equal to

$$
\begin{aligned}
\zeta & =\int_{0}^{t}(t-\tau)^{\alpha-1} \sum_{k=0}^{\infty} \frac{\alpha^{k}(\widehat{A} A)^{k}(t-\tau)^{k \alpha}}{\Gamma(\alpha k+\alpha)} \widehat{A} B\left(\alpha u(\tau)+(1-\alpha)^{C} D^{\alpha} u(\tau)\right) \mathrm{d} \tau \\
& =\lim _{N \longrightarrow \infty} \sum_{k=0}^{N}(\widehat{A} A)^{k} \widehat{A} B \int_{0}^{t}(t-\tau)^{\alpha-1} \frac{\alpha^{k}(t-\tau)^{k \alpha}}{\Gamma(\alpha k+\alpha)}\left(\alpha u(\tau)+(1-\alpha)^{C} D^{\alpha} u(\tau)\right) \mathrm{d} \tau \\
& =\lim _{N \longrightarrow \infty} \sum_{k=0}^{N}(\widehat{A} A)^{k} \widehat{A} B \beta_{k},
\end{aligned}
$$

where

$$
\beta_{k}=\int_{0}^{t}(t-\tau)^{\alpha-1} \frac{\alpha^{k}(t-\tau)^{k \alpha}}{\Gamma(\alpha k+\alpha)}\left(\alpha u(\tau)+(1-\alpha)^{C} D^{\alpha} u(\tau)\right) \mathrm{d} \tau, \quad \text { for } k=0,1, \ldots, N
$$

Writing (30) as a product, we have

$$
\zeta=\lim _{N \rightarrow \infty}\left[\begin{array}{llll}
\widehat{A} B & (\widehat{A} A) \widehat{A} B \cdots(\widehat{A} A)^{N} \widehat{A} B
\end{array}\right]\left[\begin{array}{c}
\beta_{0} \\
\beta_{1} \\
\vdots \\
\beta_{N}
\end{array}\right] .
$$

By Cayley-Hamilton theorem, any of these matrices $\widehat{A} B,(\widehat{A} A) \widehat{A} B, \ldots,(\widehat{A} A)^{N} \widehat{A} B$ is a linear combination of
$\widehat{A} B,(\widehat{A} A) \widehat{A} B, \ldots,(\widehat{A} A)^{n-1} \widehat{A} B$. Hence, $\zeta \in \operatorname{Im}\left(K_{\mathrm{ABC}}\right)$. Therefore, $R_{t} \subset \operatorname{Im}\left(K_{\mathrm{ABC}}\right)$.

In the second step, we prove that $\operatorname{Im}\left(K_{\mathrm{ABC}}\right) \subset \operatorname{Im} W_{T}$. We will show equivalently that $\left(\operatorname{Im}\left(W_{T}\right)\right)^{\perp} \subset\left(\operatorname{Im}\left(K_{\mathrm{ABC}}\right)\right)^{\perp}$, where $\left(\operatorname{Im}\left(W_{T}\right)\right)^{\perp}$ and $\left(\operatorname{Im}\left(K_{\mathrm{ABC}}\right)\right)^{\perp}$ are the orthogonal complements of $\operatorname{Im}\left(W_{T}\right)$ and $\operatorname{Im}\left(K_{\mathrm{ABC}}\right)$, respectively. We need to show that if $\zeta \in\left(\operatorname{Im}\left(W_{T}\right)\right)^{\perp}=\operatorname{Ker}\left(W_{T}^{*}\right)=$ $\operatorname{Ker}\left(W_{T}\right)$, then $\zeta \in\left(\operatorname{Im}\left(K_{\mathrm{ABC}}\right)\right)^{\perp}$. Let $\zeta \in \operatorname{Ker}\left(W_{T}\right)$. Thus, we have $\zeta^{*} W_{T} \zeta=0$, which is equivalent to 


$$
\begin{aligned}
0 & =\int_{0}^{T}(T-\tau)^{\alpha-1}\left[\zeta^{*} E_{\alpha, \alpha}\left(\alpha \widehat{A} A(T-\tau)^{\alpha}\right) \widehat{A} B\right]\left[\zeta^{*} E_{\alpha, \alpha}\left(\alpha \widehat{A} A(T-\tau)^{\alpha}\right) \widehat{A} B\right]^{*} \mathrm{~d} \tau \\
& =\int_{0}^{T}(T-\tau)^{\alpha-1}\left\|\zeta^{*} E_{\alpha, \alpha}\left(\alpha \widehat{A} A(T-\tau)^{\alpha}\right) \widehat{A} B\right\|^{2} \mathrm{~d} \tau
\end{aligned}
$$

For $\tau \in[0, T],(T-\tau)^{\alpha} \in\left[0, T^{\alpha}\right]$. Setting $z=(T-\tau)^{\alpha}$, we have

$$
\zeta^{*} E_{\alpha, \alpha}(\alpha \widehat{A} A z) \widehat{A} B=\zeta^{*} \sum_{k=0}^{\infty} \frac{\alpha^{k}(\widehat{A} A)^{k} z^{k}}{\Gamma(\alpha k+\alpha)} \widehat{A} B=0 .
$$

Differentiating $k$ times $(k=0,1,2, \ldots$,$) with respect to$ $z$ and taking the limit when $z \longrightarrow 0^{+}$implies that $\zeta^{*}(\widehat{A} A)^{k} \widehat{A} B=0$, for $k=0,1, \ldots, n-1$. It follows that

$\zeta^{*}\left[\begin{array}{llll}\widehat{A} B & (\widehat{A} A) \widehat{A} B & \cdots & (\widehat{A} A)^{n-1} \widehat{A} B\end{array}\right]=\zeta^{*} K_{\mathrm{ABC}}=0 \Longrightarrow K_{\mathrm{ABC}}^{*} \zeta=0$.

This gives us that $\zeta \in \operatorname{Ker}\left(K_{\mathrm{ABC}}^{*}\right)=\left(\operatorname{Im}\left(K_{\mathrm{ABC}}\right)\right)^{\perp}$. Therefore, $\operatorname{Im}\left(K_{\mathrm{ABC}}\right) \subset \operatorname{Im}\left(W_{T}\right)$.

In the third step, we prove that $\operatorname{Im}\left(W_{T}\right) \subset R_{t}$. Let $x_{1} \in \operatorname{Im}\left(W_{T}\right)$. Then, there exists $y$ such that $x_{1}=W_{T} y$. For every $u_{0}$ that satisfies $A x_{0}+B u_{0}=0$, we define the control $u(t)$ as

$$
\begin{gathered}
u(t)=E_{\alpha, 1}\left(-\gamma t^{\alpha}\right) u_{0}+\frac{B^{*} \widehat{A}^{*}}{(1-\alpha)} \int_{0}^{t}(t-\tau)^{\alpha-1} E_{\alpha, \alpha}\left(-\gamma(t-\tau)^{\alpha}\right) \\
E_{\alpha, \alpha}\left(\alpha A^{*} \widehat{A}^{*}(T-\tau)^{\alpha}\right) y \mathrm{~d} \tau
\end{gathered}
$$

with $\gamma=(\alpha / 1-\alpha)$. It can be shown that $D^{\alpha} u(t)$ exists and $u(t)$ satisfies in

$$
\alpha u(t)+(1-\alpha)^{C} D^{\alpha} u(t)=B^{*} \widehat{A}^{*} E_{\alpha, \alpha}\left(\alpha A^{*} \widehat{A}^{*}(T-t)^{\alpha}\right) y .
$$

Then, the solution of system (12) with $x(0)=0$ at $t=T$ is

$$
\begin{aligned}
x(T) & =\int_{0}^{T}(T-\tau)^{\alpha-1} E_{\alpha, \alpha}\left(\alpha \widehat{A} A(T-\tau)^{\alpha}\right) \widehat{A} B\left(\alpha u(\tau)+(1-\alpha)^{C} D^{\alpha} u(\tau)\right) \mathrm{d} \tau \\
& =\int_{0}^{T}(T-\tau)^{\alpha-1}\left[E_{\alpha, \alpha}\left(\alpha \widehat{A} A(T-\tau)^{\alpha}\right) \widehat{A} B\right]\left[E_{\alpha, \alpha}\left(\alpha \widehat{A} A(T-\tau)^{\alpha}\right) \widehat{A} B\right]^{*} y \mathrm{~d} \tau \\
& =W_{T}=x_{1} .
\end{aligned}
$$

Therefore, $x_{1} \in R_{t}$. This proves that $\operatorname{Im}\left(W_{T}\right) \subset R_{t}$.

Considering all three previous steps, we obtain $\operatorname{Im}\left(K_{\mathrm{ABC}}\right)=\operatorname{Im}\left(W_{T}\right)$. Since $\widehat{A} A=A \widehat{A}$, we have

$$
K_{\mathrm{ABC}}=\left[\begin{array}{cccc}
\widehat{A} & 0 & \cdots & 0 \\
0 & \widehat{A}^{2} & \cdots & 0 \\
\vdots & \vdots & \ddots & \vdots \\
0 & 0 & \cdots & \widehat{A}^{n}
\end{array}\right] K .
$$

So, $\operatorname{Im}(K)=\operatorname{Im}\left(K_{\mathrm{ABC}}\right)=\operatorname{Im}\left(W_{T}\right)$. Therefore, the Kalman matrix $K$ is full rank if and only if the Gramian matrix $W_{T}$ is nonsingular. Using Theorem 2, the proof is completed.

Using Theorem 2 and Theorem 4, we obtain the necessary and sufficient conditions for the controllability of classical linear system [38]. It should be noted that the presented criterion in Theorem 3 is similar to [14, Theorem 2] and does not depend on $\alpha$.

\section{Nonlinear System}

Consider the continuous-time nonlinear fractional system (1). Let $Q$ be the Banach space of continuous $\mathbb{R}^{n} \times \mathbb{R}^{m}$ valued function, defined on $[0, T]$ with the following norm:

$$
\|(x, u)\|_{\text {sup }}=\|x\|_{\text {sup }}+\|u\|_{\text {sup }},
$$

where $\|x\|_{\text {sup }}=\sup \{\|x(t)\|: t \in[0, T]\}$ and $\|u\|_{\text {sup }}=\sup \{\| u$ $(t) \|: t \in[0, T]\}$.

Assume that the Caputo derivatives of $u$ and $f$ exist. Then, for every $(z, v) \in Q$, a similar process of Theorem 1 can be used to show that the solution of the linear fractional system

$$
\begin{aligned}
{ }^{\mathrm{ABC}} D^{\alpha} x(t) & =A x(t)+B u(t)+f(t, z(t), v(t)), \\
x(0) & =x_{0},
\end{aligned}
$$




$$
\begin{aligned}
x(t)= & E_{\alpha, 1}\left(\alpha \widehat{A} A t^{\alpha}\right) x_{0} \\
& +\int_{0}^{t}(t-\tau)^{\alpha-1} E_{\alpha, \alpha}\left(\alpha \widehat{A} A(t-\tau)^{\alpha}\right) \widehat{A} B\left(\alpha u(\tau)+(1-\alpha)^{C} D^{\alpha} u(\tau)\right) \mathrm{d} \tau \\
& +\int_{0}^{t}(t-\tau)^{\alpha-1} E_{\alpha, \alpha}\left(\alpha \widehat{A} A(t-\tau)^{\alpha}\right) \widehat{A} \\
& \times\left(\alpha f(\tau, z(\tau), v(\tau))+(1-\alpha)^{C} D^{\alpha} f(\tau, z(\tau), v(\tau))\right) \mathrm{d} \tau .
\end{aligned}
$$

In order to analyze the controllability of nonlinear system (1), we propose the following theorem that presents sufficient conditions for the controllability of such a system.

Theorem 4. Let the continuous function $f$ with its continuous Caputo derivative satisfy the condition

$$
\lim _{\|(x, u)\|_{\text {sup }} \rightarrow \infty} \frac{\left\|\alpha f(t, x, u)+(1-\alpha)^{C} D^{\alpha} f(t, x, u)\right\|}{\|(x, u)\|_{\text {sup }}}=0,
$$

uniformly for $t \in[0, T]$. If linear system (12) is controllable, then nonlinear system (1) is controllable on $[0, T]$.

Proof. Consider the operator $P: Q \longrightarrow Q$ with $P(z, v)=(x$, $u$ ). For every $u_{0}$ that satisfies $A x_{0}+B u_{0}+f_{0}=0$, we define

$$
\begin{aligned}
u(t)= & E_{\alpha, 1}\left(-\gamma t^{\alpha}\right) u_{0} \\
& +\frac{B^{*} \widehat{A}^{*}}{(1-\alpha)} \int_{0}^{t}(t-\tau)^{\alpha-1} E_{\alpha, \alpha}\left(-\gamma(t-\tau)^{\alpha}\right) E_{\alpha, \alpha}\left(\alpha A^{*} \widehat{A}^{*}(T-\tau)^{\alpha}\right) \mathrm{d} \tau \\
& \times W_{T}^{-1}\left(x_{1}-E_{\alpha, 1}\left(\alpha \widehat{A} A T^{\alpha}\right) x_{0}-\int_{0}^{T}(T-\tau)^{\alpha-1} E_{\alpha, \alpha}\left(\alpha \widehat{A} A(T-\tau)^{\alpha}\right) \widehat{A} \times\left(\alpha f(\tau, z(\tau), v(\tau))+(1-\alpha)^{C} D^{\alpha} f(\tau, z(\tau), v(\tau))\right) \mathrm{d} \tau,\right.
\end{aligned}
$$

with $\gamma=(\alpha / 1-\alpha)$, and

$$
\begin{aligned}
x(t)= & E_{\alpha, 1}\left(\alpha \widehat{A} A t^{\alpha}\right) x_{0} \\
& +\int_{0}^{t}(t-\tau)^{\alpha-1} E_{\alpha, \alpha}\left(\alpha \widehat{A} A(t-\tau)^{\alpha}\right) \widehat{A} B\left(\alpha u(\tau)+(1-\alpha)^{C} D^{\alpha} u(\tau)\right) \mathrm{d} \tau \\
& +\int_{0}^{t}(t-\tau)^{\alpha-1} E_{\alpha, \alpha}\left(\alpha \widehat{A} A(t-\tau)^{\alpha}\right) \widehat{A} \\
& \times\left(\alpha f(\tau, z(\tau), v(\tau))+(1-\alpha)^{C} D^{\alpha} f(\tau, z(\tau), v(\tau))\right) \mathrm{d} \tau .
\end{aligned}
$$

We are going to provide the conditions for using the Schauder fixed point theorem to show that $P$ has a fixed point. According to the continuity of the Mittag-Leffler

$$
\|u(t)\| \leq c_{1}+d_{1} \sup \left\|\alpha f+(1-\alpha)^{C} D^{\alpha} f\right\|,
$$

where function and from (44), there exist positive numbers $c_{1}$ and $d_{1}$, such that for $t \in[0, T]$, we have

$$
\sup \left\|\alpha f+(1-\alpha)^{C} D^{\alpha} f\right\|=\sup \left\{\left\|\alpha f(t, z(t), v(t))+(1-\alpha)^{C} D^{\alpha} f(t, z(t), v(t))\right\|: t \in[0, T]\right\} .
$$

It should be noted that $D^{\alpha} u(t)$ exists and $u(t)$ satisfies in 


$$
\begin{aligned}
\alpha u(t) & +(1-\alpha)^{C} D^{\alpha} u(t)=B^{*} \widehat{A}^{*} E_{\alpha, \alpha}\left(\alpha A^{*} \widehat{A}^{*}(T-t)^{\alpha}\right) W_{T}^{-1} \\
& \times\left(x_{1}-E_{\alpha, 1}\left(\alpha \widehat{A} A T^{\alpha}\right) x_{0}-\int_{0}^{T}(T-\tau)^{\alpha-1} E_{\alpha, \alpha}\left(\alpha \widehat{A} A(T-\tau)^{\alpha}\right) \widehat{A} \times\left(\alpha f(\tau, z(\tau), v(\tau))+(1-\alpha)^{C} D^{\alpha} f(\tau, z(\tau), v(\tau))\right) \mathrm{d} \tau .\right.
\end{aligned}
$$

Similarly, from (45) and (48), there exist positive numbers $c_{2}$ and $d_{2}$ such that for $t \in[0, T]$,

$$
\|x(t)\| \leq c_{2}+d_{2} \sup \left\|\alpha f+(1-\alpha)^{C} D^{\alpha} f\right\| .
$$

Next, defining $c=2 \max \left\{c_{1}, c_{2}\right\}$ and $d=2 \max \left\{d_{1}, d_{2}\right\}$, we can write (46) and (49) as

$$
\begin{aligned}
& \|u(t)\| \leq \frac{1}{2}\left(c+d \sup \left\|\alpha f+(1-\alpha)^{C} D^{\alpha} f\right\|\right), \\
& \|x(t)\| \leq \frac{1}{2}\left(c+d \sup \left\|\alpha f+(1-\alpha)^{C} D^{\alpha} f\right\|\right) .
\end{aligned}
$$

From [39], since continuous function $\alpha f(t, x, u)+(1-\alpha)^{C} D^{\alpha} f(t, x, u)$ satisfies assumption (43), for every pair of positive constants $c$ and $d$, there exists a positive constant $r$ such that, if $\|(x, u)\| \leq r$, then $c+d\left\|\alpha f(t, x, u)+(1-\alpha)^{C} D^{\alpha} f(t, x, u)\right\| \leq r, \quad$ for all $t \in[0, T]$.

Next, we introduce the set $Q(r)=\{(z, v) \in Q$ : $\left.\|z\|_{\text {sup }} \leq(r / 2),\|v\|_{\text {sup }} \leq(r / 2)\right\}$. We show that $P$ maps $Q(r)$ into itself. For every $(z, v) \in Q(r)$, we have $\|z\|_{\text {sup }} \leq(r / 2)$ and $\quad\|v\|_{\text {sup }} \leq(r / 2)$. Therefore, $\quad\|(z, u)\|_{\text {sup }}=\|z\|_{\text {sup }}+$ $\|v\|_{\text {sup }} \leq(r / 2)+(r / 2)=r$, for all $t \in[0, T]$. It follows that $c+d \sup \left\|\alpha f+(1-\alpha)^{C} D^{\alpha} f\right\| \leq r$. Then, from (50) and (51), we get $\|u(t)\| \leq(r / 2)$ and $\|x(t)\| \leq(r / 2)$. Hence, $\|u\|_{\text {sup }} \leq(r / 2)$ and $\|x\|_{\text {sup }} \leq(r / 2)$. Since $\alpha f+(1-\alpha)^{C} D^{\alpha} f$ is continuous, the operator $P$ is continuous. It should be noted that $Q(r)$ is closed, bounded, and convex, so by the Schauder fixed point theorem [40, Theorem 4.14], $P$ has a fixed point $(z, v) \in Q(r)$, such that $P(z, v)=(z, v) \equiv(x, u)$. Therefore, we rewrite (17) and (18) as

$$
\begin{aligned}
u(t)= & E_{\alpha, 1}\left(-\gamma t^{\alpha}\right) u_{0} \\
& +\frac{B^{*} \widehat{A}^{*}}{(1-\alpha)} \int_{0}^{t}(t-\tau)^{\alpha-1} E_{\alpha, \alpha}\left(-\gamma(t-\tau)^{\alpha}\right) E_{\alpha, \alpha}\left(\alpha A^{*} \widehat{A}^{*}(T-\tau)^{\alpha}\right) \mathrm{d} \tau \\
& \times W_{T}^{-1}\left(x_{1}-E_{\alpha, 1}\left(\alpha \widehat{A} A T^{\alpha}\right) x_{0}-\int_{0}^{T}(T-\tau)^{\alpha-1} E_{\alpha, \alpha}\left(\alpha \widehat{A} A(T-\tau)^{\alpha}\right) \widehat{A} \times\left(\alpha f(\tau, x(\tau), u(\tau))+(1-\alpha)^{C} D^{\alpha} f(\tau, x(\tau), u(\tau))\right) \mathrm{d} \tau,\right.
\end{aligned}
$$

and

$$
\begin{aligned}
& x(t)=E_{\alpha, 1}\left(\alpha \widehat{A} A t^{\alpha}\right) x_{0} \\
& +\int_{0}^{t}(t-\tau)^{\alpha-1} E_{\alpha, \alpha}\left(\alpha \widehat{A} A(t-\tau)^{\alpha}\right) \widehat{A} B\left(\alpha u(\tau)+(1-\alpha)^{C} D^{\alpha} u(\tau)\right) \mathrm{d} \tau \\
& +\int_{0}^{t}(t-\tau)^{\alpha-1} E_{\alpha, \alpha}\left(\alpha \widehat{A} A(t-\tau)^{\alpha}\right) \\
& \times \widehat{A}\left(\alpha f(\tau, x(\tau), u(\tau))+(1-\alpha)^{C} D^{\alpha} f(\tau, x(\tau), u(\tau))\right) \mathrm{d} \tau, \\
& c_{1}=a_{1}+\frac{T^{\alpha} \alpha^{-1} a_{2} a_{3}}{1-\alpha}\left\|B^{*}\right\|\left\|\widehat{A}^{*}\right\|\left\|W_{T}^{-1}\right\|\left(\left\|x_{1}\right\|+a_{4}\right), \\
& d_{1}=\frac{\left(T^{\alpha} \alpha^{-1} a_{3}\right)^{2} a_{2}}{1-\alpha}\left\|B^{*}\right\|\left\|W_{T}^{-1}\right\|\|\widehat{A}\|^{2} \\
& c_{2}=a_{4}+T^{\alpha} \alpha^{-1} a_{3}^{2}\|\widehat{A}\|^{2}\|B\|^{2}\left\|W_{T}^{-1}\right\|\left(\left\|x_{1}\right\|+a_{4}\right), \\
& d_{2}=\left(T^{\alpha} \alpha^{-1}\right)^{2} a_{3}^{3}\|\widehat{A}\|^{3}\|B\|^{2}\left\|W_{T}^{-1}\right\|+T^{\alpha} \alpha^{-1} a_{3}\|\widehat{A}\|,
\end{aligned}
$$

which shows that $x(t)$ is the solution of nonlinear system (1). Now, setting $t=T$ in (54), we get $x(T)=x_{1}$, so nonlinear system (1) is controllable.

Proposition 1. The values of positive numbers $c_{1}, d_{1}, c_{2}$, and $d_{2}$ used in Theorem 4 are calculated by 
where

$$
\begin{aligned}
& a_{1}=\sup _{t \in[0, T]}\left\|E_{\alpha, 1}\left(-\gamma t^{\alpha}\right) u_{0}\right\|, \\
& a_{2}=\sup _{t \in[0, T]}\left\|E_{\alpha, \alpha}\left(-\gamma(T-t)^{\alpha}\right)\right\|, \\
& a_{3}=\sup _{t \in[0, T]}\left\|E_{\alpha, \alpha}\left(\alpha \widehat{A} A(T-t)^{\alpha}\right)\right\|, \\
& a_{4}=\sup _{t \in[0, T]}\left\|E_{\alpha, 1}\left(\alpha \widehat{A} A t^{\alpha}\right) x_{0}\right\| .
\end{aligned}
$$

Proof. According to (53) and (56), we can write

$$
\begin{aligned}
\|u(t)\| \leq & a_{1}+\frac{1}{1-\alpha}\left\|B^{*}\right\|\left\|\widehat{A}^{*}\right\| t^{\alpha} \alpha^{-1} a_{2} a_{3}\left\|W_{T}^{-1}\right\| \\
& \times\left(\left\|x_{1}\right\|+a_{4}+T^{\alpha} \alpha^{-1} a_{3}\|\widehat{A}\| \sup \left\|\alpha f+(1-\alpha)^{C} D^{\alpha} f\right\|\right) \\
& \leq a_{1}+\frac{T^{\alpha} \alpha^{-1} a_{2} a_{3}}{1-\alpha}\left\|B^{*}\right\|\left\|\widehat{A}^{*}\right\|\left\|W_{T}^{-1}\right\|\left(\left\|x_{1}\right\|+a_{4}\right) \\
& +\frac{\left(T^{\alpha} \alpha^{-1} a_{3}\right)^{2} a_{2}}{1-\alpha}\left\|B^{*}\right\|\left\|\widehat{A}^{*}\right\|\left\|W_{T}^{-1}\right\|\|\widehat{A}\| \sup \left\|\alpha f+(1-\alpha)^{C} D^{\alpha} f\right\| .
\end{aligned}
$$

Defining

$$
\begin{aligned}
& c_{1}=a_{1}+\frac{T^{\alpha} \alpha^{-1} a_{2} a_{3}}{1-\alpha}\left\|B^{*}\right\|\left\|\widehat{A}^{*}\right\|\left\|W_{T}^{-1}\right\|\left(\left\|x_{1}\right\|+a_{4}\right), \\
& d_{1}=\frac{\left(T^{\alpha} \alpha^{-1} a_{3}\right)^{2} a_{2}}{1-\alpha}\left\|B^{*}\right\|\left\|W_{T}^{-1}\right\|\|\widehat{A}\|^{2},
\end{aligned}
$$

yields

$$
\|u(t)\| \leq c_{1}+d_{1} \sup \left\|\alpha f+(1-\alpha)^{C} D^{\alpha} f\right\|
$$

Similarly, it follows from (54) that

$$
\|x(t)\| \leq a_{4}+T^{\alpha} \alpha^{-1} a_{3}\|\widehat{A}\|\|B\|\left\|\alpha u+(1-\alpha)^{C} D^{\alpha} u\right\|_{\text {sup }}+T^{\alpha} \alpha^{-1} a_{3}\|\widehat{A}\| \sup \left\|\alpha f+(1-\alpha)^{C} D^{\alpha} f\right\| .
$$

Using (48) yields

$$
\begin{aligned}
\| \alpha u(t) & +(1-\alpha)^{C} D^{\alpha} u(t)\|\leq\| B^{*}\|\| \widehat{A}^{*}\left\|a_{3}\right\| W_{T}^{-1} \| \\
& \times\left(\left\|x_{1}\right\|+a_{4}+T^{\alpha} \alpha^{-1} a_{3}\|\widehat{A}\| \sup \left\|\alpha f+(1-\alpha)^{C} D^{\alpha} f\right\|\right) .
\end{aligned}
$$

$$
\begin{aligned}
\|x(t)\| \leq & a_{4}+T^{\alpha} \alpha^{-1} a_{3}^{2}\|\widehat{A}\|\|B\|\left\|B^{*}\right\|\left\|\widehat{A}^{*}\right\|\left\|W_{T}^{-1}\right\|\left(\left\|x_{1}\right\|+a_{4}\right) \\
& +\left(\left(T^{\alpha} \alpha^{-1}\right)^{2} a_{3}^{3}\|\widehat{A}\|^{2}\|B\|\left\|B^{*}\right\|\left\|\widehat{A}^{*}\right\|\left\|W_{T}^{-1}\right\|+T^{\alpha} \alpha^{-1} a_{3}\|\widehat{A}\|\right) \sup \left\|\alpha f+(1-\alpha)^{C} D^{\alpha} f\right\| .
\end{aligned}
$$

Then, defining

$$
\begin{aligned}
& c_{2}=a_{4}+T^{\alpha} \alpha^{-1} a_{3}^{2}\|\widehat{A}\|^{2}\|B\|^{2}\left\|W_{T}^{-1}\right\|\left(\left\|x_{1}\right\|+a_{4}\right), \\
& d_{2}=\left(T^{\alpha} \alpha^{-1}\right)^{2} a_{3}^{3}\|\widehat{A}\|^{3}\|B\|^{2}\|\| W_{T}^{-1}\left\|+T^{\alpha} \alpha^{-1} a_{3} \widehat{A}\right\|,
\end{aligned}
$$

we derive

$$
\|x(t)\| \leq c_{2}+d_{2} \sup \left\|\alpha f+(1-\alpha)^{C} D^{\alpha} f\right\| .
$$

It should be noted that due to the existence of $x /(t)$ or $u \prime(t)$ or both of them in the calculation of the Caputo derivative of $f(t, x(t), u(t))$, we present the following proposition, which is an appropriate tool for examining condition (43).

Proposition 2. Assume that the notations $a_{2}, a_{3}$, and $a_{4}$ are described in Proposition 1. Then, the derivatives of $x(t)$ and $u(t)$ exist and are bounded. 
Proof. According to (53), we can write

$$
\begin{aligned}
u \prime(t)= & E_{\alpha, 1}^{\prime}\left(-\gamma t^{\alpha}\right) u_{0} \\
& +\frac{B^{*} \widehat{A}^{*}}{(1-\alpha)} \int_{0}^{t}\left((\alpha-1)(t-\tau)^{\alpha-2} E_{\alpha, \alpha}\left(-\gamma(t-\tau)^{\alpha}\right)+(t-\tau)^{\alpha-1} E_{\alpha, \alpha}^{\prime}\left(-\gamma(t-\tau)^{\alpha}\right)\right) \\
& \times E_{\alpha, \alpha}\left(\alpha A^{*} \widehat{A}^{*}(T-\tau)^{\alpha}\right) \mathrm{d} \tau \\
& \times W_{T}^{-1}\left(x_{1}-E_{\alpha, 1}\left(\alpha \widehat{A} A T^{\alpha}\right) x_{0}-\int_{0}^{T}(T-\tau)^{\alpha-1} E_{\alpha, \alpha}\left(\alpha \widehat{A} A(T-\tau)^{\alpha}\right) \times \widehat{A}\left(\alpha f(\tau, x(\tau), u(\tau))+(1-\alpha)^{C} D^{\alpha} f(\tau, x(\tau), u(\tau))\right) \mathrm{d} \tau\right.
\end{aligned}
$$

Setting we have

$$
\begin{aligned}
& a_{5}=\sup _{t \in[0, T]}\left\|E_{\alpha, 1}^{\prime}\left(-\gamma t^{\alpha}\right) u_{0}\right\|, \\
& a_{6}=\sup _{t \in[0, T]}\left\|E_{\alpha, \alpha}^{\prime}\left(-\gamma(T-t)^{\alpha}\right)\right\|,
\end{aligned}
$$

$$
\begin{aligned}
\|u \prime(t)\| \leq a_{5} & +\frac{1}{1-\alpha}\left\|B^{*}\right\|\left\|\widehat{A}^{*}\right\|\left(t^{\alpha-1} a_{2}+t^{\alpha} \alpha^{-1} a_{6}\right) a_{3}\left\|W_{T}^{-1}\right\| \\
& \times\left(\left\|x_{1}\right\|+a_{4}+T^{\alpha} \alpha^{-1} a_{3}\|\widehat{A}\| \sup \left\|\alpha f+(1-\alpha)^{C} D^{\alpha} f\right\|\right) \\
\leq & a_{5}+\frac{\left(T^{\alpha-1} a_{2}+T^{\alpha} \alpha^{-1} a_{6}\right) a_{3}}{1-\alpha}\left\|B^{*}\right\|\left\|\widehat{A}^{*}\right\|\left\|W_{T}^{-1}\right\|\left(\left\|x_{1}\right\|+a_{4}\right) \\
& +\frac{\left(T^{2 \alpha-1} \alpha^{-1} a_{2}+T^{2 \alpha} \alpha^{-2} a_{6}\right) a_{3}^{2}}{1-\alpha}\left\|B^{*}\right\|\left\|\widehat{A}^{*}\right\|\left\|W_{T}^{-1}\right\|\|\widehat{A}\| \sup \left\|\alpha f+(1-\alpha)^{C} D^{\alpha} f\right\| .
\end{aligned}
$$

\section{Defining}

$$
\begin{aligned}
& c_{3}=a_{5}+\frac{\left(T^{\alpha-1} a_{2}+T^{\alpha} \alpha^{-1} a_{6}\right) a_{3}}{1-\alpha}\left\|B^{*}\right\|\left\|\widehat{A}^{*}\right\|\left\|W_{T}^{-1}\right\|\left(\left\|x_{1}\right\|+a_{4}\right), \\
& d_{3}=\frac{\left(T^{2 \alpha-1} \alpha^{-1} a_{2}+T^{2 \alpha} \alpha^{-2} a_{6}\right) a_{3}^{2}}{1-\alpha}\left\|B^{*}\right\|\left\|W_{T}^{-1}\right\|\|\widehat{A}\|^{2},
\end{aligned}
$$

yields

$$
\|u(t)\| \leq c_{3}+d_{3} \sup \left\|\alpha f+(1-\alpha)^{C} D^{\alpha} f\right\| .
$$

$$
\begin{aligned}
x^{\prime}(t)= & E_{\alpha, 1}^{\prime}\left(\alpha \widehat{A} A t^{\alpha}\right) x_{0} \\
& +\int_{0}^{t}\left((\alpha-1)(t-\tau)^{\alpha-2} E_{\alpha, \alpha}\left(\alpha \widehat{A} A(t-\tau)^{\alpha}\right)+(t-\tau)^{\alpha-1} E_{\alpha, \alpha}^{\prime}\left(\alpha \widehat{A} A(t-\tau)^{\alpha}\right)\right) \\
& \times \widehat{A} B\left(\alpha u(\tau)+(1-\alpha)^{C} D^{\alpha} u(\tau)\right) \mathrm{d} \tau \\
& +\int_{0}^{t}\left((\alpha-1)(t-\tau)^{\alpha-2} E_{\alpha, \alpha}\left(\alpha \widehat{A} A(t-\tau)^{\alpha}\right)+(t-\tau)^{\alpha-1} E_{\alpha, \alpha}^{\prime}\left(\alpha \widehat{A} A(t-\tau)^{\alpha}\right)\right) \\
& \times \widehat{A}\left(\alpha f(\tau, x(\tau), u(\tau))+(1-\alpha)^{C} D^{\alpha} f(\tau, x(\tau), u(\tau))\right) \mathrm{d} \tau .
\end{aligned}
$$


Setting

$$
\begin{aligned}
& a_{7}=\sup _{t \in[0, T]}\left\|E_{\alpha, \alpha}^{\prime}\left(\alpha \widehat{A} A(T-t)^{\alpha}\right)\right\|, \\
& a_{8}=\sup _{t \in[0, T]}\left\|E_{\alpha, 1}^{\prime}\left(\alpha \widehat{A} A t^{\alpha}\right) x_{0}\right\|,
\end{aligned}
$$

$$
\begin{aligned}
\left\|x^{\prime}(t)\right\| \leq a_{7} & +\left(T^{\alpha-1} a_{3}+T^{\alpha} \alpha^{-1} a_{8}\right)\|\widehat{A}\|\|B\|\left\|\alpha u+(1-\alpha)^{C} D^{\alpha} u\right\|_{\text {sup }} \\
& +\left(T^{\alpha-1} a_{3}+T^{\alpha} \alpha^{-1} a_{8}\right)\|\widehat{A}\| \sup \left\|\alpha f+(1-\alpha)^{C} D^{\alpha} f\right\| .
\end{aligned}
$$

Then, using (61) yields

we obtain

$$
\begin{aligned}
\|x \prime(t)\| \leq & a_{7}+\left(T^{\alpha-1} a_{3}+T^{\alpha} \alpha^{-1} a_{8}\right) a_{3}\|\widehat{A}\|^{2}\|B\|^{2}\left\|W_{T}^{-1}\right\|\left(\left\|x_{1}\right\|+a_{4}\right) \\
& +\left(\left(T^{2 \alpha-1} \alpha^{-1} a_{3}+T^{2 \alpha} \alpha^{-2} a_{8}\right) a_{3}^{2}\|\widehat{A}\|^{3}\|B\|^{2}\left\|W_{T}^{-1}\right\|+\left(T^{\alpha-1} a_{3}+T^{\alpha} \alpha^{-1} a_{8}\right)\|\widehat{A}\|\right) \\
& \times \sup \left\|\alpha f+(1-\alpha)^{C} D^{\alpha} f\right\| .
\end{aligned}
$$

Defining

$$
\begin{aligned}
& c_{4}=a_{7}+\left(T^{\alpha-1} a_{3}+T^{\alpha} \alpha^{-1} a_{8}\right) a_{3}\|\widehat{A}\|^{2}\|B\|^{2}\left\|W_{T}^{-1}\right\|\left(\left\|x_{1}\right\|+a_{4}\right), \\
& d_{4}=\left(T^{2 \alpha-1} \alpha^{-1} a_{3}+T^{2 \alpha} \alpha^{-2} a_{8}\right) a_{3}^{2}\|\widehat{A}\|^{3}\|B\|^{2}\left\|W_{T}^{-1}\right\|+\left(T^{\alpha-1} a_{3}+T^{\alpha} \alpha^{-1} a_{8}\right)\|\widehat{A}\|,
\end{aligned}
$$

we derive

$$
\|x \prime(t)\| \leq c_{4}+d_{4} \sup \left\|\alpha f+(1-\alpha)^{C} D^{\alpha} f\right\| .
$$

\section{Numerical Examples}

In this section, we utilize some examples to illustrate the validity of our results.

Example 1. We consider the following linear system:

$$
\begin{aligned}
{ }^{\mathrm{ABC}} D^{\alpha} x(t) & =A x(t)+B u(t), \\
x(0) & =x_{0},
\end{aligned}
$$

where $\alpha=(1 / 3), t \in[0,2]$, and

$$
A=\left[\begin{array}{cc}
2 & -1 \\
-1 & 2
\end{array}\right] \text {, }
$$

$$
B=\left[\begin{array}{c}
\frac{2}{3} \\
-\frac{1}{3}
\end{array}\right] \text {. }
$$

From (21), the controllability Gramian matrix is

$$
W_{2}=\int_{0}^{2}(2-\tau)^{\alpha-1}\left[E_{\alpha, \alpha}\left(\alpha \widehat{A} A(2-\tau)^{\alpha}\right) \widehat{A} B\right]\left[E_{\alpha, \alpha}\left(\alpha \widehat{A} A(2-\tau)^{\alpha}\right) \widehat{A} B\right]^{*} \mathrm{~d} \tau
$$

where

$$
E_{\alpha, \alpha}\left(\alpha \widehat{A} A(2-\tau)^{\alpha}\right)=\left[\begin{array}{ll}
\sum_{k=0}^{\infty} \frac{(2-\tau)^{2 k \alpha}}{\Gamma((2 k+1) \alpha)} & \sum_{k=0}^{\infty} \frac{(2-\tau)^{(2 k+1) \alpha}}{\Gamma((2 k+2) \alpha)} \\
\sum_{k=0}^{\infty} \frac{(2-\tau)^{(2 k+1) \alpha}}{\Gamma((2 k+2) \alpha)} & \sum_{k=0}^{\infty} \frac{(2-\tau)^{2 k \alpha}}{\Gamma((2 k+1) \alpha)}
\end{array}\right] .
$$


Then, defining $c_{1}=\sum_{k=0}^{\infty}\left((2-\tau)^{2 k \alpha} / \Gamma((2 k+1) \alpha)\right)$ and $c_{2}=\sum_{k=0}^{\infty}\left((2-\tau)^{(2 k+1) \alpha} / \Gamma((2 k+2) \alpha)\right)$ yields

$$
E_{\alpha, \alpha}\left(\alpha \widehat{A} A(2-\tau)^{\alpha}\right)=\left[\begin{array}{ll}
c_{1} & c_{2} \\
c_{2} & c_{1}
\end{array}\right] \text {. }
$$

Therefore, (78) becomes

$$
W_{2}=\int_{0}^{2}(2-\tau)^{\alpha-1}\left[\begin{array}{cc}
c_{2}^{2} & c_{1} c_{2} \\
c_{1} c_{2} & c_{1}^{2}
\end{array}\right] \mathrm{d} \tau
$$

which is nonsingular.
Also, the Kalman matrix $K=1 / 3\left[\begin{array}{cc}2 & 5 \\ -1 & -4\end{array}\right]$ is full rank. Hence, from Theorem 2 or Theorem 3, system (76) with control $u(t)$ defined in (22) is controllable. We consider $x_{1}(t)$ and $x_{2}(t)$ as the solutions of system (76) for initial conditions $x_{1}(0)$ and $x_{2}(0)$, respectively. Here

$$
\begin{aligned}
& x_{1}(t)=\left[\begin{array}{l}
x_{11}(t) \\
x_{21}(t)
\end{array}\right], \\
& x_{2}(t)=\left[\begin{array}{l}
x_{12}(t) \\
x_{22}(t)
\end{array}\right], \\
& x_{1}(0)=\left[\begin{array}{l}
1 \\
0
\end{array}\right], \\
& x_{2}(0)=\left[\begin{array}{c}
-2 \\
0
\end{array}\right] .
\end{aligned}
$$

The control $u(t)$ steers system (76) from $x_{1}(0)$ and $x_{2}(0)$ to $x_{1}(2)$ and $x_{2}(2)$, respectively, where

$$
\begin{aligned}
& x_{1}(2)=\left[\begin{array}{l}
5 \\
1
\end{array}\right], \\
& x_{2}(2)=\left[\begin{array}{l}
4 \\
2
\end{array}\right] .
\end{aligned}
$$

Now, consider the following system:

$$
\begin{aligned}
{ }^{\mathrm{ABC}} D^{\alpha} x(t) & =A x(t)+B u(t)+\left[\begin{array}{c}
0 \\
\sqrt{t}
\end{array}\right], \\
x(0) & =x_{0},
\end{aligned}
$$

where $A, B, \alpha$, and $t$ are as above. According to the above description, this system is also controllable. Using Matlab, the trajectories of solutions are depicted in Figures 1 and 2.

Example 2. We consider the nonlinear system as follows:

$$
\begin{aligned}
{ }^{\mathrm{ABC}} D^{\alpha} x(t) & =A x(t)+B u(t)+f(t, x(t)), \\
x(0) & =x_{0},
\end{aligned}
$$

where $f(t, x(t))=\left[\begin{array}{c}0 \\ \left(1 / 1+x_{1}^{2}(t)\right)\end{array}\right]$ and $A, B, \alpha$, and $t$ are the same as in the Example 1. The Caputo derivative of $f$ is

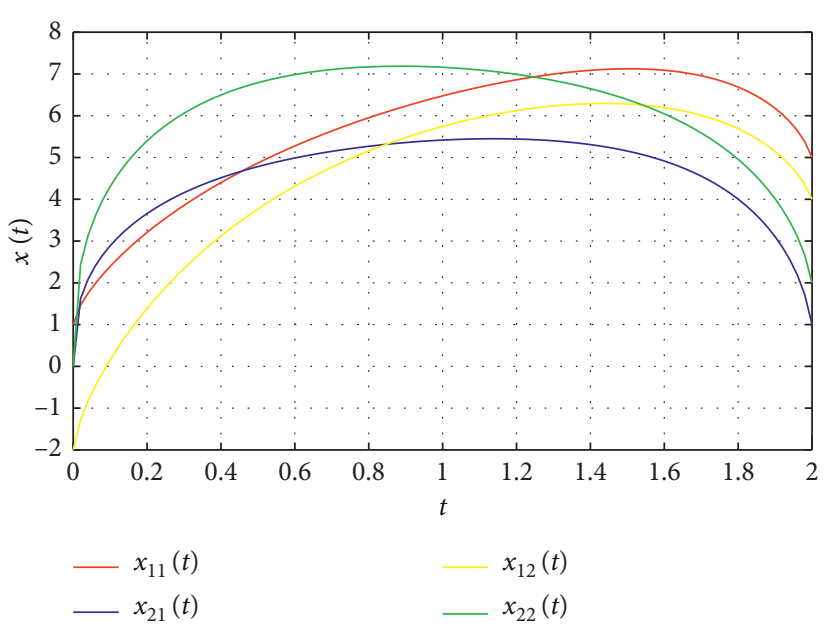

FIgURE 1: The trajectories of linear system (76).

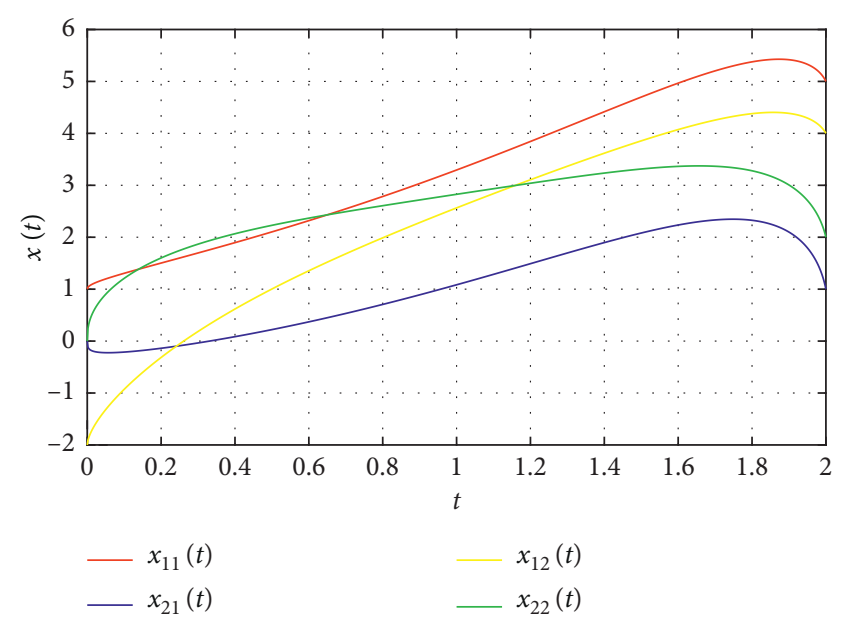

Figure 2: The trajectories of linear system (84).

$$
{ }^{C} D^{\alpha} f(t, x(t))=\frac{1}{\Gamma(2 / 3)}\left[\int_{0}^{t}-2 x_{1}(\tau) x_{1}^{\prime}(\tau) /\left(1+x_{1}^{2}(\tau)\right)^{2}(t-\tau)^{(1 / 3)} \mathrm{d} \tau\right] .
$$

From Proposition 2, $\alpha f+(1-\alpha)^{C} D^{\alpha} f$ satisfies condition (43). Also, according to Example 1, the linear system is controllable. Therefore, nonlinear system (85) is controllable.

\section{Conclusion}

In this paper, we obtained the solution of the linear fractional system involving the Atangana-Baleanu derivative with the Caputo approach. According to the definition of controllability, we presented some necessary and sufficient conditions for the controllability of such a system based on the controllability Gramian matrix and the Kalman matrix. Using the Schauder fixed point theorem and the obtained results for the linear system, we also established sufficient conditions for the controllability of the nonlinear system. The reliability of the analytical process used in our work has been checked by numerical examples. 


\section{Data Availability}

No data were used to support this study.

\section{Conflicts of Interest}

The authors declare that there are no conflicts of interest regarding the publication of this paper.

\section{References}

[1] V. G. Jensen and G. V. Jeffreys, Mathematical Methods in Chemical Engineering, Academic Press, London, UK, 1977.

[2] Y. A. Rossikhin and M. V. Shitikova, "Application of fractional derivatives to the analysis of damped vibrations of viscoelastic single mass systems," Acta Mechanica, Springer, vol. 120, no. 1-4, pp. 109-125, 1997.

[3] S. H. Weinberg, "Membrane capacitive memory alters spiking in neurons described by the fractional-order hodgkin-huxley model," PLoS One, vol. 10, no. 5, Article ID e0126629, 2015.

[4] R. L. Magin, Fractional Calculus in Bioengineering, Begell House Redding, CT, USA, 2006.

[5] C. K. Kwuimy, G. Litak, and C. Nataraj, "Nonlinear analysis of energy harvesting systems with fractional order physical properties, Nonlinear Dynamics," Springer, vol. 80, no. 1, pp. 491-501, 2015.

[6] N. Laskin, "Fractional market dynamics," Physica A: Statistical Mechanics and Its Applications, Elsevier, vol. 287, no. 3-4, pp. 482-492, 2000.

[7] A. A. Kilbas, H. M. Srivastava, and J. J. Trujillo, Theory and Applications of Fractional Differential Equations, Elsevier, Amsterdam, The Netherlands, 2006.

[8] W. H. Deng, "Smoothness and stability of the solutions for nonlinear fractional differential equations," Nonlinear Analysis: Theory, Methods \& Applications, vol. 72, no. 3-4, pp. 1768-1777, 2010.

[9] C. Hwang and Y.-C. Cheng, "A numerical algorithm for stability testing of fractional delay systems," Automatica, vol. 42 , no. 5, pp. 825-831, 2006.

[10] M. S. Tavazoei and M. Haeri, "A note on the stability of fractional order systems," Mathematics and Computers in Simulation, vol. 79, no. 5, pp. 1566-1576, 2009.

[11] M. S. Tavazoei, M. Haeri, S. Bolouki, and M. Siami, "Stability preservation analysis for frequency-based methods in numerical simulation of fractional order systems," SIAM Journal on Numerical Analysis, vol. 47, no. 1, pp. 321-338, 2009.

[12] J. Klamka, Controllability of Dynamical Systems, Kluwer Academic, Dordrecht, Netherlands, 1993.

[13] J. Klamka, "Controllability of semilinear systems with multiple variable delays in control, Mathematics," Multidisciplinary Digital Publishing Institute, vol. 8, no. 11, pp. 1-9, 2020.

[14] S. Buedo-Fernández and J. J. Nieto, "Basic control theory for linear fractional differential equations with constant coefficients," Frontiers in Physics, Frontiers, vol. 8, p. 377, 2020.

[15] K. Balachandran, M. Matar, and J. J. Trujillo, "Note on controllability of linear fractional dynamical systems," Journal of Control and Decision, vol. 3, no. 4, pp. 267-279, 2016.

[16] B. Sikora and J. Klamka, "Constrained controllability of fractional linear systems with delays in control," Systems \& Control Letters, vol. 106, pp. 9-15, 2017.

[17] K. Balachandran and V. Govindaraj, "Numerical controllability of fractional dynamical systems," Optimization, vol. 63, no. 8, pp. 1267-1279, 2014.
[18] K. Balachandran, J. Y. Park, and J. J. Trujillo, "Controllability of nonlinear fractional dynamical systems," Nonlinear Analysis: Theory, Methods and Applications, vol. 75, no. 4, pp. 1919-1926, 2012.

[19] X.-L. Ding, J. J. Nieto, and J. J. Nieto, "Controllability of nonlinear fractional delay dynamical systems with prescribed controls," Nonlinear Analysis: Modelling and Control, vol. 23, no. 1, pp. 1-18, 2018.

[20] N. I. Mahmudov, "Variational approach to finite-approximate controllability of sobolev-type fractional systems," Journal of Optimization Theory and Applications, vol. 184, no. 2, pp. 671-686, 2020.

[21] I. Podlubny, Fractional Differential Equations, Academic Press, New York, USA, 1999.

[22] S. G. Samko, A. A. Kilbas, and O. I. Marichev, Fractional Integrals and Derivatives-Theory and Applications, Gordon and Breach, Yverdonn, Switzerland, 1993.

[23] M. Caputo and M. Fabrizio, "A new definition of fractional derivative without singular kernel," Progress.in Fractional. Differentiation Applications, vol. 1, no. 2, pp. 73-85, 2015.

[24] T. A. Yildız, A. Jajarmi, B. Yıldız, and D. Baleanu, "New aspects of time fractional optimal control problems within operators with nonsingular kernel, Discrete \& Continuous Dynamical Systems-S," American Institute of Mathematical Sciences, vol. 13, no. 3, pp. 407-428, 2020.

[25] A. Atangana and D. Baleanu, "New fractional derivatives with nonlocal and non-singular kernel: theory and application to heat transfer model," Thermal Science, vol. 20, no. 2, pp. 763-769, 2016.

[26] K. Diethelm, R. Garrappa, A. Giusti, and M. Stynes, "Why fractional derivatives with nonsingular kernels should not be used," Fractional Calculus and Applied Analysis, vol. 23, no. 3, pp. 610-634, 2020.

[27] A. Giusti, "A comment on some new definitions of fractional derivative," Nonlinear Dynamics, vol. 93, no. 3, pp. 1757-1763, 2018.

[28] E. Capelas de Oliveira, S. Jarosz, and J. Vaz, "On the mistake in defining fractional derivative using a non-singular kernel," arXiv e-prints, 2019, https://arxiv.org/abs/1912.04422.

[29] M. D. Ortigueira, V. Martynyuk, M. Fedula, and J. T. Machado, "The failure of certain fractional calculus operators in two physical models," Fractional Calculus and Applied Analysis, vol. 22, no. 2, pp. 255-270, 2019.

[30] A. Atangana and J. F. Gómez-Aguilar, "Fractional derivatives with no-index law property: application to chaos and statistics," Chaos, Solitons and Fractals, vol. 114, pp. 516-535, 2018.

[31] J. Sabatier, "Fractional-order derivatives defined by continuous kernels: are they really too restrictive?" Fractal and Fractional, vol. 4, no. 3, p. 40, 2020.

[32] D. Baleanu, "Comments on: "The failure of certain fractional calculus operators in two physical models," in Fractional Calculus and Applied Analysis, M. Ortigueira, V. Martynyuk, M. Fedula, and J. A. T. Machado, Eds., vol. 23, no. 1, pp. 292-297, 2020.

[33] A. Atangana and E. F. D. Goufo, "Some misinterpretations and lack of understanding in differential operators with no singular kernels," Open Physics, vol. 18, no. 1, pp. 594-612, 2020.

[34] J. Hristov, "Derivatives with non-singular kernels from the caputo-fabrizio definition and beyond: appraising analysis with emphasis on diffusion models," Frontiers in Fractional Calculus, vol. 1, pp. 235-295, 2017. 
[35] V. Govindaraj, M. Malik, and R. K. George, "Trajectory controllability of fractional dynamical systems," Journal of Control and Decision, vol. 4, no. 2, pp. 114-130, 2017.

[36] A. Erdélyi, W. Magnus, F. Oberhettinger, and F. G. Tricomi, Higher Transcendental Functions, Vol. 3, McGraw-Hill, New York, USA, 1955.

[37] Y. Chen, H. S. Ahn, and D. Xue, "Robust controllability of interval fractional order linear time invariant systems," Signal Processing, vol. 10, no. 86, pp. 2794-2802, 2006.

[38] R. E. Kalman, P. L. Falb, and M. A. Arbib, Topics in Mathematical System Theory, McGraw-Hill, New York, NY, USA, 1969.

[39] J. P. Dauer, "Nonlinear perturbations of quasi-linear control systems," Journal of Mathematical Analysis and Applications, vol. 54, no. 3, pp. 717-725, 1976.

[40] R. P. Agarwal, M. Meehan, and D. O'regan, Fixed Point Theory and Applications, Cambridge University Press, Cambridge, UK, 2001. 